\title{
Mecanismos que garantizan la inscripción temprana del recién nacido en el Registro del Estado Familiar como forma de tutelar su derecho a la identidad en la ciudad de Santa Ana
}

\author{
Juan Carlos Orellana Villalobos ${ }^{1}$ \\ Karina Beatriz Orellana Villalobos ${ }^{2}$ \\ Docentes investigadores, Facultad de Ciencias y Humanidades \\ Universidad Católica de El Salvador, El Salvador
}

Fecha de recepción: 06-12-2019

Fecha de aceptación: $31-01-2020$

\section{Resumen}

El nacimiento de una persona conlleva una serie de obligaciones, siendo la primera de ellas su inscripción en el Registro del Estado Familiar correspondiente a su lugar de nacimiento, o en el lugar donde los padres tengan su domicilio. Esto constituye un derecho de todos los individuos, ya que con esto al recién nacido se le reconoce personalidad jurídica. Al no inscribírsele, se le considera como no sujeto de derecho. Esta situación impide al niño el acceso a todos los demás derechos humanos fundamentales.

La investigación desarrollada en el departamento de Santa Ana se fundamentó en información documental disponible y en entrevistas realizadas a los actores involucrados en la temática, a fin de ejecutar un análisis sobre la efectividad de los mecanismos que garantizan la inscripción temprana de los recién nacidos en el Registro del Estado Familiar. Esta problemática es trascendental, debido a que el derecho a la identidad e identificación es fundamental para la persona.

En ese sentido, el Estado es el primero que debe garantizar que las personas recién nacidas sean identificadas oportunamente, mediante la aplicación de procedimientos ágiles y sencillos. Es esta entidad la que debe implementar y fortalecer vínculos, entre las instituciones gubernamentales y no gubernamentales implicadas en el proceso; así como crear mecanismos idóneos que lo permitan. Se pudo constatar que los procesos administrativos actuales, desarrollados por algunas instituciones públicas de salud, no son los suficientemente efectivos en el cumplimiento de este propósito. Es necesario crear o mejorar los ya existentes; además de contar con un procedimiento idóneo y completo de inscripción temprana del recién nacido.

Palabras clave: Derecho a la identidad, inscripción temprana, asiento de nacimiento, derecho al nombre, partida de nacimiento, alcaldía municipal.

\begin{abstract}
The birth of a person carries a series of obligations, being the first of them their registration in the Family Status Registry corresponding to their place of birth, or in the place where the parents have their domicile. This constitutes a right of all individuals, since with this the newborn is recognized as a legal personality. By not registering him or her. He or she is considered as not subject to rights. This situation prevents the child from accessing all the other fundamental human rights.

The research developed in the department of Santa Ana was based on available documentary information and interviews conducted with actors involved in the subject, in order to implement an analysis about the effectiveness of the mechanisms that guarantee the early registration of the newborns in the Family Status Registry. This problem is momentous, inasmuch as the right to identity and identification is fundamental for the person

In this sense, the State is the first that has to guarantee that newborns are identified in a timely manner, through the application of agile and simple procedures. It is this entity the one that must implement and strengthen bonds between the governmental and non-governmental institutions involved in the process; as well as creating suitable mechanisms that allow it. It was proved that the current administrative processes, developed by some public health institutions, are not sufficiently effective in the achievement of this purpose. It is necessary to create or improve existing ones in addition to having a suitable and complete procedure for the early registration of the newborn.
\end{abstract}

Key words: Right to identity, early registration, birth place, right to name, birth record, municipal city hall.

1. Licenciado en Ciencias Jurídicas; email: j.orellana@catolica.edu.sv; ORCID: https://orcid.org/0000-0002-5328-1825

2. Licenciada en Ciencias Jurídicas; email: karina.orellana@catolica.edu.sv; ORCID: https://orcid.org/0000-0002-4117-6665 


\section{Introducción}

El derecho a la identidad constituye un atributo esencial de la persona, ya que por medio de este resultan y se fortalecen los derechos y deberes que se originan dentro de la vida en sociedad. En doctrina se conocen dos aspectos esenciales de este derecho de identidad: el estático, que se refiere a los elementos que identifican a la persona (nombre y apellido, domicilio, nacionalidad); y el dinámico, referido a todos los vínculos de tipo familiar, religioso y asistencial, que conforman la identidad a lo largo de la vida del sujeto. La prueba de la identidad es lo que se denomina identificación.

La Constitución de la República de El Salvador, en el artículo 36 inciso tercero estatuye: “(...) Toda persona tiene derecho a tener un nombre que la identifique. La ley secundaria regulará esta materia (...)". De esta forma, se establece el nombre como elemento de la personalidad, permitiendo la individualización de la persona, y del cual se derivan todos los demás derechos, obligaciones y beneficios indispensables para tener una vida digna.

Esta norma constitucional se desarrolla a través de diversas leyes, tales como: La Ley del Nombre de la Persona Natural, que en sus artículos 1 y 4 consagran el derecho al nombre, considerándolo como el elemento que encabeza la partida de nacimiento; la Ley Transitoria del Registro del Estado Familiar, disponiendo en el artículo 1 el establecimiento de un régimen para registrar, conservar y facilitar la localización y consulta de información relacionada al estado familiar de las personas.

Por su parte, la Ley de Protección Integral de la Niñez y Adolescencia (LEPINA) ${ }^{3}$ en el artículo 73 determina que:

Las niñas, niños y adolescentes tienen derecho a la identidad y a los elementos que la constituyen, especialmente al nombre, la nacionalidad, a su relación paterna y materna filiales y a la obtención de documentos públicos de identidad (...) Es obligación del Estado crear programas para que las instituciones públicas competentes garanticen la identidad de toda niña, niño y adolescente.

La Declaración Universal de los Derechos $\mathrm{Hu}-$ manos, en su artículo 6 establece que, "Todo ser humano tiene derecho, en todas partes, al reconocimiento de su personalidad jurídica"; mientras que El Salvador, con la ratificación de la Convención sobre los Derechos del Niño, asumió el compromiso de adoptar aquellas medidas legislativas y políticas necesarias para dar efectividad a los derechos de los niños y adolescentes. Lo anterior tiene la finalidad de asegurar la protección integral de los infantes, disponiendo en su artículo 8, la obligación de los Estados de garantizar el derecho del niño a preservar su identidad. Esto se evidencia especialmente en el artículo 7 número 1:

3. Dentro del documento, los autores se referirán a este documento mediante su acrónimo. 
El niño será inscrito inmediatamente después de su nacimiento y tendrá derecho, desde que nace, a su nombre; a adquirir una nacionalidad y, en la medida de lo posible, a conocer a sus padres y a ser cuidado por ellos.

Desde el momento en el que nace, el niño necesita una identidad. Es decir, ser inscrito en el Registro del Estado Familiar de su lugar de nacimiento o en el lugar donde tengan domicilio sus padres. El registro del nacimiento es obligatorio y, además, un derecho de todos los individuos, ya que reconoce su personalidad jurídica y la garantiza frente a terceros.

\section{Registro de identidad}

El Estado tiene la obligación ineludible de garantizar el derecho a la identidad personal, debiendo crear e implementar los mecanismos administrativos idóneos de registros, que faciliten y simplifiquen la inscripción del recién nacido. La inscripción del nacimiento es el acto administrativo-registral, a través del cual no solo se reconoce legalmente la existencia de una persona, y a la que se le otorga un acta de nacimiento (o partida de nacimiento); sino que, además, indica que ésta posee un nombre por el cual será distinguido de por vida, permitiendo su identificación e individualización. Lleva a la persona a pertenecer a una familia, a una comunidad y a una nación. Todo ello se hace efectivo a través de la inscripción del nacimiento en la Oficina del Registro del Estado Familiar de manera oportuna o de forma extemporánea.
En el país, los mecanismos y procedimientos administrativos existentes no son lo suficientemente eficaces para tal propósito, dejando a la persona en un estado de inexistencia legal y vulnerando uno de sus derechos fundamentales. La violación al derecho a la identidad de los niños y adolescentes genera consecuencias jurídicas y sociales graves e irreversibles. Al negárseles su inscripción, no tiene existencia legal y se les impide el acceso a servicios básicos, tales como: ingresar al sistema educativo; la acreditación de sus estudios, acceso a programas de salud pública o seguridad social, entre otros. Tal situación, en muchas ocasiones, afecta a su descendencia, pues cuando estos niños llegan a la adultez no poseen documento de identidad, haciendo difícil la inscripción de sus hijos, y perpetuando los problemas jurídicos de identidad.

Ante esto, surgió la pregunta de investigación: ¿Cuál es el nivel de efectividad de los mecanismos que garantizan la inscripción temprana del recién nacido en el Registro del Estado Familiar con el objeto de tutelar su derecho a la identidad en la ciudad de Santa Ana?

Para dar respuesta a ello, se analizó el rol que desempeñan tanto las instituciones privadas como estatales involucradas para asegurar el derecho a la identidad e identificación de los niños y adolescentes. Se indagó sobre los mecanismos, estrategias, programas y procedimientos que se llevan a cabo, a fin de conocer las funciones y perspectivas de las instituciones encargadas de velar por el cumplimiento de ese 
derecho. Esto con el propósito de determinar si las soluciones o alternativas de dichos mecanismos son accesibles y efectivos; o, por el contrario, se restringe o se hace nugatorio el derecho a una inscripción temprana. Se aportan también, recomendaciones que coadyuven a mejorar la aplicación de los mismos, resaltando la importancia de que la comunidad en general conozca dichos mecanismos para acceder a ellos de manera eficiente.

\section{Metodología}

La investigación fue de tipo evaluativa, fundamentada en la legislación existente sobre la materia, doctrina y experiencia de las diferentes instituciones encargadas de salvaguardar el derecho a la identidad.

Los sujetos de estudio fueron representantes y personal de instituciones públicas y privadas, involucradas en velar por garantizar el derecho a la identidad e identificación en la ciudad de Santa Ana (ver figura 1).

La técnica utilizada para recopilar la información necesaria fue la entrevista estructurada. Se elaboró una guía de entrevista con preguntas abiertas pertinentes al tema en estudio, para luego comparar, contrastar y analizar las respuestas obtenidas.

\section{Resultados}

\section{a. Registro del Estado Familiar de la Alcal- día Municipal de Santa Ana}

Las personas entrevistadas dentro de esta entidad expresaron que, hace aproximadamen- te cinco años, existía un convenio de coordinación suscrito por la Alcaldía Municipal de Santa Ana, el Registro Nacional de la Persona Natural (RNPN) y el Hospital San Juan de Dios de ese departamento. Este establecía un procedimiento de captación de datos de recién nacidos, en donde se trabajaba coordinadamente con personal de las áreas: Jurídica, Trabajo Social y Enfermería del hospital; y con empleados del Registro Nacional de Personas Naturales.

Según este mecanismo, los empleados pertenecientes al RNPN capturaban en formularios los datos sobre el recién nacido y sus padres; posteriormente presentaban dicha documentación junto con las huellas plantares al Registro del Estado Familiar de la Alcaldía de Santa Ana, a efecto que se ingresará la información directamente a la plataforma digital de dicho Registro.

Con esto se lograba que las madres, al darse de alta del hospital, no se retiraran sin haber inscrito el nacimiento de su hijo; asegurando así la inscripción temprana del recién nacido. Esto daba como resultado que una vez se practicaba el asentamiento, inmediatamente se podía extender gratuitamente la certificación de partida de nacimiento.

De acuerdo a las declaraciones, diariamente se realizaba un aproximado de 15 a 20 inscripciones de recién nacidos; certificando por lo menos que el 90\% de ellos se asentaran legalmente. La acción incluía tanto al departamento de Santa Ana (70\% de los nacidos), como a los de otros departamentos (Ahuachapán y Son- 


\begin{tabular}{|c|c|c|}
\hline Entidad & Funciones de los entrevistados & $\begin{array}{c}\text { Cantidad de } \\
\text { sujetos abordados }\end{array}$ \\
\hline $\begin{array}{c}\text { Registro del Estado Familiar } \\
\text { (REF) de la Alcaldía Municipal } \\
\text { de Santa Ana }\end{array}$ & $\begin{array}{l}\text { Jefe del Registro del Estado Fa- } \\
\text { miliar y encargados de la Oficina } \\
\text { de Atención Temprana (OAT)*. }\end{array}$ & 4 \\
\hline $\begin{array}{l}\text { Hospital Regional del Instituto } \\
\text { Salvadoreño del Seguro Social } \\
\text { (ISSSS), Santa Ana }\end{array}$ & $\begin{array}{l}\text { Jefe de la Unidad de Afiliación, } \\
\text { empleados del área de Trabajo } \\
\text { Social y Enfermería. }\end{array}$ & 3 \\
\hline Procuraduría Auxiliar de Santa Ana & $\begin{array}{l}\text { Empleado de la Unidad de de- } \\
\text { fensa de la familia }\end{array}$ & 1 \\
\hline $\begin{array}{l}\text { Hospital Nacional San Juan de } \\
\text { Dios, Santa Ana }\end{array}$ & $\begin{array}{l}\text { Asesor Jurídico y personal de } \\
\text { Trabajo Social y Enfermería. }\end{array}$ & 3 \\
\hline $\begin{array}{c}\text { Registro Nacional de las Personas } \\
\text { Naturales (RNPN), Santa Ana }\end{array}$ & Empleado de la institución & 1 \\
\hline Centros hospitalarios privados & $\begin{array}{l}\text { Encargados Administrativos y } \\
\text { Enfermería }\end{array}$ & 4 \\
\hline \multicolumn{2}{|c|}{ Total } & 16 \\
\hline
\end{tabular}

Figura 1. Cuadro resumen de la muestra.

sonate), pues era permitido el asentamiento de los nacimientos de otros municipios, siempre y cuando los padres así lo decidieran, aun teniendo domicilio en esos lugares. Esto último, estaba amparado de conformidad con lo expuesto en el artículo 28 de la Ley Transitoria del Registro del Estado Familiar y en los Regímenes Patrimoniales del Matrimonio. No obstante, algunas personas no tenían conocimiento de este proceso, por lo que era necesaria la divulgación y la publicidad del mismo, a fin de considerarlo como un proceso obligatorio.

La actividad y el rol de cooperación y coordinación entre las tres instituciones, derivadas del documento suscrito, fue efectiva; ya que prácticamente la madre, el padre o ambos no necesitaban apersonarse a la oficina del Registro del Estado Familiar de la alcaldía a brindar los datos del nacimiento. En esencia, el proceso permitía registrar legalmente el nacimiento y extender a corto plazo, sin ningún costo pecuniario, la certificación de partida de nacimiento. Sin embargo, esta coordinación no fue continua en el tiempo, debido a diversas circunstancias que los entrevistados desconocen, pues para esas fechas no laboraban en el Registro del Estado Familiar. Actualmente ya no existe este mecanismo de colaboración, pues primero se retiró el personal del RNPN y luego el personal del Registro del Estado Familiar, que había sido delegado para tal efecto. 
En consecuencia, ahora la madre o ambos padres deben acudir directamente a la oficina del Registro del Estado Familiar a proporcionar los datos del recién nacido, y realizar el procedimiento necesario para asentar a su hijo. Además de solicitar por primera vez la certificación de partida de nacimiento del niño, deben cancelar la tasa municipal establecida para tal efecto.

\section{b. Hospital Nacional San Juan de Dios de Santa Ana}

Por su parte, el personal entrevistado del Hospital Nacional San Juan de Dios de Santa Ana manifestó que, durante el año 2019, no existió trabajo ni coordinación para facilitar la inscripción temprana del recién nacido con personal de la oficina del Registro del Estado Familiar y delegados del RNPN. Sin embargo, durante el año 2018, el Hospital se coordinaba con Ciudad Mujer para los casos de madres que no poseían ningún tipo de registro de nacimiento, a efecto que les apoyaran en el proceso de búsqueda de inscripción en el RNPN; o en su caso, contactar a la Procuraduría General de la República para solicitar asesoría jurídica y asentar con prontitud el nacimiento de los niños.

En su momento, cuando existían delegados del RNPN, estos hacían una especie de pre- asentamiento del recién nacido, que luego remitían a la oficina del Registro del Estado Familiar para la inscripción definitiva del nacimiento; pero desde hace varios años, cesó el apoyo al hospital por parte de dicha institución.

Los sujetos entrevistados expresaron que aún, cuando en el hospital no se cuenta con una oficina de atención temprana de inscripción de recién nacido, el área de Servicio Social se encarga de concientizar a las madres sobre la necesidad y el deber de inscribir al recién nacido en el Registro del Estado Familiar. Además, antes del año 2009, solamente expedían los plantares del recién nacido para entregarlos a dicha oficina; pero, en la actualidad, y en cumplimiento al artículo 75 de la Ley de Protección Integral de la Niñez y Adolescencia (LEPINA), se elabora una ficha médica de control por cada recién nacido.

Esta ficha contiene entre otros datos: nombre del recién nacido -con ello se garantiza el derecho a un nombre propio según la Ley del Nombre, pues años atrás solamente se hacían constar los apellidos del recién nacido-; nombres e información de la madre o padres, registro de vacunas, nombre del médico y enfermera que atendió el parto; huellas plantares y la firma de la madre o ambos padres.

Se conoció también que, desde el mes de enero de 2017 para llevar un mejor control, registro e identificación del recién nacido en todas sus etapas de desarrollo, el hospital asigna a cada niño un número único de nacimiento, denominado Código Único al Nacer $(\mathrm{CUN})^{4}$. Este número no puede modificarse y evita posibles adopciones irregulares o suplantaciones de estado familiar. Este código tiene el propósito de que se tenga acceso digitalmente el archivo de

4. Es el sistema registral mediante el cual, el Hospital le asigna a cada recién nacido un número para su identificación; similar al número del Documento Único de Identidad (DUI) de los adultos. Este registro se introduce en el sistema del nosocomio e incluye los datos de: fecha de nacimiento del niño, nombres de los padres, número del expediente, entre otros. Los autores harán referencia a él mediante su acrónimo. 
los datos del menor, compartidos en el sistema hospitalario, si los padres del niño acuden a cualquier institución de salud o protección pública. Es menester que los padres se aseguren de llevar anotado el CUN, ya que este debe ser presentado en el establecimiento donde se realizarán los controles del niño y en los hospitales públicos, cuando amerite atención médica hospitalaria.

Además, explicaron que, con la adopción de este mecanismo, los datos de nacimiento y demás información del recién nacido jamás podrán desaparecer, puesto que se encuentra en formato físico y en soporte digital. En años anteriores, los datos y plantares del niño se conservaban únicamente por un período de cinco a diez años y; posteriormente, se descartaban.

En los casos de madres que no poseen asiento de partida de nacimiento, y en consecuencia tampoco documento de identidad, para efectos del registro médico del recién nacido se le identifica por medio de testigos y mediante la firma de un acta, creada por el Área Jurídica del hospital. Este documento tiene la finalidad de que, al dársele de alta, se cuente con información completa respecto a la filiación materna del recién nacido. Según los entrevistados, el número de niños que nacen mensualmente en ese nosocomio es de aproximadamente 400 a 500; aunque aclararon que no envían informes de datos sobre nacimientos al Registro del Estado Familiar.

\section{c. Hospital Regional del Instituto Salvadore- ño del Seguro Social de Santa Ana}

El personal del Hospital Regional del Instituto Salvadoreño del Seguro Social de Santa Ana (ISSS), desde hace aproximadamente dos años, trabaja conjuntamente con el Registro del Estado Familiar de la Alcaldía Municipal de Santa Ana. Para tal acción se ha adecuado provisionalmente un espacio físico dentro del hospital, en donde funciona la Oficina de Atención Temprana (OAT). En este mecanismo intervienen personal del referido hospital (Trabajo Social, Enfermería y Unidades Administrativas), así como empleados de dicha entidad.

El proceso inicia desde que la mujer se encuentra en estado de embarazo hasta su alumbramiento, pues se le imparten charlas no solamente sobre el cuidado de su bebé, sino también sobre la obligación y necesidad de inscribir el nacimiento de su hijo recién nacido, con el fin de garantizar el derecho a la identidad e identificación. Diariamente, dos empleados del Registro del Estado Familiar son enviados al ISSS, a efecto de capturar la información de los recién nacidos e inscribirlos. Estas personas se encuentran en el lugar desde las 10:00 a.m. hasta horas de la tarde, con el objeto de recibir de la madre o de ambos padres los datos y documentos relativos al nacimiento.

Esta información se vacía en un formulario avalado por REF, con el fin de realizar una "reserva de folio",

\footnotetext{
5. Entiéndase como el mecanismo por medio del cual los empleados solicitan de forma correlativa a la oficina registral, la reserva en el Sistema Informático de Folios y Números de partidas de nacimientos de la Alcaldía Municipal de Santa Ana.w
} 
puedan apersonarse posteriormente al Registro del Estado Familiar. Este documento debe ser firmado por la madre o por quien brinde los datos. Los padres disponen de un plazo de 24 a 72 horas para refrendar el asiento de partida de nacimiento original definitiva. Si los padres no se presentan a la entidad para realizar este último paso, el niño no estará inscrito legalmente.

Para afiliar al recién nacido como beneficiario del Seguro Social y, en consecuencia, brindarle atención médica pediátrica inmediata, es suficiente que cuente con este documento provisional; mientras los padres realizan el trámite para el asentamiento definitivo. Una vez obtenida la partida de nacimiento, los padres deben presentarse a las oficinas administrativas de filiación del ISSS para darle legalidad al trámite.

Los entrevistados mencionaron que no se asigna un CUN a cada recién nacido, tal como lo hace el Hospital San Juan de Dios; y que el documento provisional de reserva de folio solo aplica para madres o padres que deseen que su hijo sea asentado en el municipio de Santa Ana. Si los padres optan por asentarlo en un municipio distinto, únicamente se les entregan los documentos de huellas plantares, a fin de que el interesado informe e inscriba el nacimiento en el municipio de su respectivo domicilio. Asimismo, el ISSS lleva su propio archivo sobre el número de nacimientos y afiliaciones de recién nacidos, afirmando que mensualmente nacen aproximadamente de 300 a 400 niños, que son hijos de empleados públicos o privados.

\section{d. Hospitales privados}

Los entrevistados que laboran en el Hospital Cader de Santa Ana manifestaron que, las madres que dan a luz completan un formulario de registro de nacimiento denominado Certificado de Nacimiento. Dentro de este se hace constar: nombre del recién nacido, nombre de la madre o de ambos padres; domicilio, fecha, hora y lugar del nacimiento; peso, talla, perímetro cefálico, nombres del médico ginecólogo y enfermera que atendió el parto, entre otros insumos. Los datos de identificación de los padres se toman de sus respectivos documentos de identidad. En el caso que el padre se encuentre fuera del país, no se consigna esta filiación, puesto que es necesaria su presencia física con el fin de reconocer la paternidad voluntariamente del recién nacido.

Este documento, que lleva las huellas plantares, es entregado a la madre, explicándole la obligación de inscribir el nacimiento de su hijo en el Registro del Estado Familiar competente. Sin embargo, expresaron que en algunos municipios diferentes al de Santa Ana -como en el caso del Registro del Estado Familiar de Chalchuapa- solicitan datos adicionales que, según ellos, deben constar en el Certificado de Nacimiento. Esto trae como resultado que la madre o padre deba regresar al hospital para modificar o ampliar dicho certificado para posteriormente presentarlo al Registro y realizar la inscripción del recién nacido. 
Por otra parte, explicaron que no se asigna un CUN como se realiza en el Hospital San Juan de Dios, sino que el Certificado de Nacimiento lleva asignado el número del expediente médico de la madre, que fue atendida en el nosocomio. Este documento se conserva en los archivos del hospital por un plazo máximo de cinco años; transcurrido este tiempo, se depura. En el caso de extravío de los plantares o Certificado de Nacimiento, se extiende una copia al interesado legítimo, adjuntando una nota explicativa de dicho extravío, siempre y cuando el expediente se encuentre en los archivos del hospital. Asimismo, expresaron que no existe trabajo coordinado con el REF y el RNPN, a efecto de coadyuvar al proceso de asentamiento del recién nacido.

Por otro lado, la información obtenida por personal del Hospital Centro Médico de Santa Ana manifestó que, en los casos de nacimientos, extienden a la madre un Registro de Nacimiento con los datos de identificación del recién nacido y huellas plantares; nombres de los padres conforme a sus documentos únicos de identidad, entre otra información. Dicho documento tiene un número asignado por el hospital para un mayor control. Los plantares se archivan temporalmente por un término de dos semanas, aproximadamente.

Agregaron que el número de recién nacidos en ese hospital oscilan entre 5 a 6 mensuales, y que informan semanalmente al Ministerio de Salud este dato para fines estadísticos. Sin embargo, no existe coordinación alguna con el REF de Santa Ana, a efecto de enviarles información relacionada con los natalicios y su inscripción correspondiente. Por su parte, solamente les hacen saber a los padres del recién nacido la necesidad y obligación de inscribir a su hijo en el registro.

\section{e. Procuraduría General de la República y \\ Registro Nacional de Personas Naturales}

Por su parte, los entrevistados de la Agencia Auxiliar de la Procuraduría General de la República y del Registro Nacional de Personas Naturales (RNPN) en el departamento de Santa Ana expresaron que, actualmente, no participan activamente en los procesos de inscripción temprana de los recién nacidos que implementan el Hospital San Juan de Dios y el Hospital Regional del Instituto Salvadoreño del Seguro Social.

No obstante, aclararon que se está trabajando para que durante el 2020, mediante el RNPN, se suscriban convenios con los nosocomios y la Alcaldía Municipal de Santa Ana, a fin de coordinar programas y estrategias que coadyuven a la mejora de los procesos ya existentes, o creación de otros de similar naturaleza.

\section{Discusión}

Garantizar el derecho a la identidad e identificación es fundamental para la persona, puesto que se le reconoce su individualidad como sujeto de derechos que deben ser respetados tanto por el Estado, la sociedad y la familia a la que pertenece. Este reconocimiento como principio 
de la existencia legal, se materializa al momento de asentar al recién nacido, y se prueba jurídicamente con la Certificación de Partida de Nacimiento, en la cual consta no solamente el nombre de la persona, sino también su filiación materna y paterna, según sea el caso.

Es, por tanto, un imperativo para el Estado asegurar este derecho de la persona desde el momento de su nacimiento, mediante la creación e implementación de mecanismos idóneos que -de manera expedita, no burocrática- permitan hacer efectivo este derecho; evitando su vulneración. En este sentido, es oportuno mencionar la existencia, en años anteriores, de un proceso que facilitaba la inscripción temprana del recién nacido en el Hospital Nacional San Juan de Dios frente al Registro del Estado Familiar de la Alcaldía Municipal de Santa Ana. Cabe resaltar que, mientras se llevó a cabo este mecanismo de tarea conjunta, hubo resultados muy satisfactorios, en cuanto a facilitar y garantizar a los padres la inscripción de su recién nacido en un plazo de tiempo relativamente corto; así como de llevar a cabo el deber del Estado de velar por los derechos fundamentales del niño.

No obstante, es lamentable que actualmente no existe ningún mecanismo de inscripción temprana de los recién nacidos en ese nosocomio, quedando a discreción de los padres del menor de edad hacerlo dentro del plazo legal que estipula la ley 6 , o bien, hacerlo extempo- ráneamente. $\mathrm{O}$, en casos más graves, omitir negligentemente tal registro de nacimiento, con las consecuencias nefastas que esto acarrea en los ámbitos social, familiar, cultural y legal del niño.

\section{Proyección a futuro}

Ante la falta de inscripción temprana del recién nacido e inexistencia de procedimientos que coadyuven a paliar esta situación, el 20 de mayo del 2019, la Alcaldía Municipal de Santa Ana y el Hospital Nacional San Juan de Dios del mismo municipio celebraron un convenio de cooperación continua. En él se establece que, de conformidad al artículo 36 de la Constitución de la República de El Salvador, el Estado es el garante de velar y hacer efectivo el derecho a la identidad, mediante la prestación de servicios de calidad; que redunden en beneficio de la población, particularmente de los recién nacidos. Ante esto se crea el programa denominado "Inscripción Temprana de la Niña y el Niño en el Registro del Estado Familiar y Menores del municipio de Santa Ana”.

El documento tiene por objeto fortalecer el rol de estas instituciones en cuanto a tutelar el derecho a la identidad del recién nacido. Entre las responsabilidades adquiridas a futuro por ambas instituciones, se contempla la ejecución de actividades de promoción y educación de los usuarios del Hospital Nacional San Juan de Dios, a fin que conozcan los beneficios de ese

\footnotetext{
6. De conformidad con el artículo 28, inciso 1 de la Ley Transitoria del Registro del Estado Familiar y Regímenes Patrimoniales del Matrimonio, en El Salvador, los padres de los recién nacidos tienen un plazo de 90 días, posteriores al nacimiento, para realizar el asentamiento.
} 
programa y la necesidad de la inscripción temprana del niño como una obligación ineludible de los padres.

Con esto se daría mayor cobertura en los servicios de inscripción en los diferentes municipios de Santa Ana; o por lo menos, se les podría remitir a los padres a las respectivas oficinas registrales, aun cuando no exista acuerdo entre el hospital y las mismas. Al mismo tiempo, también se podrían realizar las respectivas inscripciones de las defunciones ocurridas en el nosocomio y la expedición de otras certificaciones relacionadas.

Se pretende habilitar un espacio físico de carácter permanente dentro de las instalaciones del hospital en donde funcione la Oficina de Atención Temprana (OAT). Dicha dependencia tendría acceso a internet para operar el sistema de inscripción temprana y la plataforma del Registro del Estado Familiar para realizar la expedición inmediata de la correspondiente partida de nacimiento. Con estas acciones, mecanismos y otras estrategias se espera mejorar la calidad y celeridad en la prestación del servicio de registro temprano de nacimientos, y así reducir el problema de la falta de inscripción de recién nacidos con los perjuicios que de ello resultan.

Por su parte, el Hospital Regional del Instituto Salvadoreño del Seguro Social, firmó el 2 de mayo de 2019, un convenio de cooperación mutua, de naturaleza semejante al firmado con el Hospital San Juan de Dios de Santa Ana. En él se estipula que los suscriptores del mismo trabajarán coordinada y progresivamente en el fortalecimiento y mejoramiento de la Oficina de Atención Temprana (OAT) que ya existe. Según los entrevistados, esto comenzaría a ejecutarse a principios del año 2020.

Ellos agregaron que el documento suscrito ha tenido el apoyo del Fondo de las Naciones Unidas para la infancia (UNICEF), invirtiendo y facilitando recursos económicos destinados a desarrollar la infraestructura, adquirir equipo informático y demás recursos materiales requeridos para mejorar y ampliar los servicios de la OAT. Al respecto, refirieron que lo que se pretende realizar a corto plazo con esta oficina es crear un minidistrito del municipio, con el objeto de ampliar la cobertura de los servicios brindados por el Registro, y la descentralización de los mismos; en donde, además de capturar datos del recién nacido, se extienda la correspondiente certificación de asiento de partida de nacimiento.

Todos estos esfuerzos pretenden reajustar procedimientos que, actualmente, se desarrollan en los municipios de Santa Tecla (La Libertad) y San Salvador (San Salvador), a fin de descentralizar la carga laboral de las oficinas del Registro del Estado Familiar.

\section{Esfuerzos actuales}

Por otra parte, es significativo el desarrollo de un proyecto incipiente para facilitar el asentamiento de los recién nacidos en el Hospital Regional del Seguro Social de Santa Ana, en 
coordinación con la oficina del Registro del Estado Familiar de Santa Ana. No obstante, pese a los buenos resultados inscribiendo provisionalmente, mediante la figura de "reserva de folio" a los recién nacidos en ese nosocomio, todavía no es suficiente. Este proceso no ahorra el hecho que los padres del menor deban apersonarse a la oficina del Registro del Estado Familiar para realizar el trámite.

Si al momento de proporcionar los datos en la OAT ubicada en el hospital, el sistema informático en línea de la oficina fuese capaz de procesar la información del asiento de nacimiento definitivo del niño, se reducirían significativamente el tiempo que lleva el proceso, así como los gastos pecuniarios de los padres del recién nacido, especialmente cuando estos son de escasos recursos económicos. Asimismo, se evitaría el problema de la falta de comparecencia de los padres, ya que algunos por ignorancia o malentendidos asumen que, al proporcionar los datos de su hijo en la OAT, éste ya se encuentra legal y definitivamente inscrito. Incluso, algunos no se apersonan a firmar en el Registro del Estado Familiar, por considerar esto un trámite burocrático. Esto último es un problema complicado, puesto que, existiendo una reserva de folio solicitada por la OAT, si la madre, padre o ambos no se presentan a compadecer y firmar, el folio reservado se anula, suspende o sustituye por otro asiento dentro del Registro.

En otro orden de ideas, es rescatable y conveniente el mecanismo de la asignación de un número único de nacimiento (CUN) a cada recién nacido. Si bien esto solo es implementado por el Hospital San Juan de Dios de Santa Ana como una forma de identificar inequívocamente sus datos personales durante el resto de su vida, debería pensarse en ingresar esta información a un sistema digital, concentrado a cargo del RNPN y del Ministerio de Salud.

Esto podría ser obligatorio y extensivo para todas las instituciones hospitalarias, públicas o privadas, como un mecanismo de salvaguarda, protección y control de la información del nacimiento de todo niño; especialmente en los casos de extravío de ficha médica y plantares, o para diligencias judiciales subsidiarias de posesión de estado familiar, en caso se haya omitido la inscripción de la persona, entre otros. Con este procedimiento podrían evitarse futuros problemas legales o ilícitos que atenten contra el derecho a la identidad e identificación de la persona. Por otra parte, una forma de motivar a los padres para que realicen la inscripción temprana de su hijo recién nacido en el Registro del Estado Familiar, es no cobrar ninguna tasa registral al momento de extender, por primera vez, la certificación de partida de nacimiento. Pese a ello, actualmente los padres deben cancelar los aranceles correspondientes, luego de efectuado el asiento respectivo.

\section{Unanimidad}

En lo que respecta a los hospitales privados, los entrevistados manifestaron que no existe coordinación de ninguna clase en lo referente 
a información de nacimientos e inscripción de los niños, pues -a su entender- en dichos nosocomios cada mujer que da a luz tiene la obligación de asentar a su hijo, presentándose directamente a la oficina de Registro correspondiente. De ahí que resulta conveniente tanto, estandarizar la información contenida en la ficha médica y en las huellas plantares de nacimiento que lleva cada institución hospitalaria pública o privada, como capacitar jurídicamente al personal del Registro del Estado Familiar; ya que algunos de ellos realizan observaciones burocráticas y exigen requisitos adicionales no los contemplados en el artículo 65 de la LEPINA. Implementar estas recomendaciones evitarían la pérdida de tiempo y dinero; postergación o desmotivación por parte de los padres para inscribir al niño.

Así también, llama la atención que, tratándose del caso de los hospitales privados, no existe ningún tipo de entendimiento ni cooperación con la oficina del Registro del Estado Familiar, y mucho menos con el RNPN para propiciar el registro del nacimiento de los recién nacidos. No solo queda a discreción o negligencia de los padres, sino que tampoco se remite o comparte ningún tipo de información con las instituciones públicas relacionadas al tema. Estos datos, al escapar del control del Estado, podrían ocasionar resultados gravosos y posibles actos constitutivos de delitos, como la trata de personas, falsos partos, suplantación, entre otros.

Es indispensable que exista una cooperación y coordinación efectiva entre las entidades públi- cas e instituciones hospitalarias privadas, debiendo las primeras iniciar los acercamientos y contactos necesarios para dar cumplimiento al artículo 28 de la Ley Transitoria del Registro del Estado Familiar. Es necesario, en este caso, que al personal del Registro del Estado Familiar se le brinde colaboración y acceso a la información de los hospitales privados, a efecto de cumplir con la normativa legal. La misma situación aplica para los hospitales San Juan de Dios e Instituto Salvadoreño del Seguro Social, en el sentido que no se cumple con la remisión de la información. Además, es importante que el RNPN, Alcaldía Municipal de Santa Ana e instituciones hospitalarias coordinen esfuerzos para solicitar apoyo financiero y técnico a entidades nacionales o internacionales, que velan por la protección de los derechos de la niñez.

Entre las dificultades que se presentan, en un futuro cercano para las instituciones hospitalarias públicas, es establecer el procedimiento a seguir para asentar a niños nacidos en el municipio de Santa Ana, cuyos padres no residen ahí y desean inscribir a sus hijos en los respectivos municipios de donde son oriundos. En este caso, por no ser competencia del municipio de Santa Ana, es evidente la necesidad de realizar convenios con otros municipios para tener presencia en esas instituciones hospitalarias, y trabajar de forma coordinada con el RNPN. Entonces, es indispensable la intervención activa de esta entidad por ser una de las principales garantes del derecho a la identidad de cada persona. 
En conclusión, la falta u omisión de la inscripción temprana del recién nacido no solamente vulnera el derecho a la identidad de la persona, sino que también conlleva graves consecuencias en cuanto al no reconocimiento de sus derechos fundamentales (educación, salud, entre otros), impidiéndole el desempeño efectivo dentro de la sociedad. Esta situación también da lugar a situaciones irregulares y comisión de delitos como el tráfico de niños, suplantación de estado familiar, alteración de la identidad personal, abuso de menores, prostitución infantil y las llamadas adopciones irregulares o ilegales.

Todas estas situaciones no deben tolerarse ni permitirse por parte del Estado, en donde su finalidad está orientada al beneficio, desarrollo y protección de la persona humana, en todos los estadios de su vida desde su concepción. En virtud de esto, es imperativo e insoslayable para todos los actores (Estado, familia, la comunidad y la sociedad en general) crear y ejecutar y vigilar, de forma coordinada y progresiva, mecanismos idóneos para la protección integral de los derechos de los recién nacidos.

Por otro lado, también es necesaria la divulgación y promoción de los mecanismos, tanto de los ya existentes como de los que a futuro se puedan implementar, tanto para educar a la población en general como para crear conciencia sobre la responsabilidad de los padres, en cuanto a la necesidad e importancia de la inscripción temprana de sus hijos en el Registro del Estado Familiar.

\section{Referencias}

Asamblea Nacional de las Naciones Unidas (20 de noviembre de 1989). Convención sobre los derechos del niño.

Barillas, S. (2004). Apuntes sobre Derecho Procesal de Familia. El Salvador: Editorial Lis.

Buaiz, Y. E. (2013). Ley de Protección Integral de la Niñez y Adolescencia comentada de El Salvador. Libro Primero. San Salvador: Consejo Nacional de la Judicatura.

Buitrago, A. y Bonilla, E. (1996). Manual de Derecho de Familia. El Salvador.

Bossert, G. A. y Zannoni, E. (2001). Manual de Derecho de Familia. Buenos Aires.

Mendoza, R. (2010). Código de Familia. San Salvador: Editorial Jurídica Salvadoreña.

Mendoza, R. (2010). Constitución de la República. San Salvador: Editorial Jurídica Salvadoreña.

Mendoza, R. (2010). Ley del Nombre de la Persona Natural. San Salvador: Editorial Jurídica Salvadoreña. 
Mendoza, R. (2010). Ley Procesal de Familia. San Salvador, El Salvador: Editorial Jurídica Salvadoreña.

Mendoza, R. (2010). Ley de Protección de la Niñez y Adolescencia. San Salvador: Editorial Jurídica Salvadoreña.

UNICEF (2002). Sesión Especial en favor de la infancia. Recuperado de https://www.unicef.org/ spanish/why/why_special_session.html

UNICEF (2002). Un Mundo Apropiado para los Niños. Recuperado de https://www.unicef.org/ spanish/publications/index_45482.html 